\title{
Use of anti-dementia drugs and delayed care home placement: an observational study
}

\author{
Emad Salib, ${ }^{1}$ Jessica Thompson ${ }^{2}$
}

The Psychiatrist (2011), 35, 384-388, doi: 10.1192/pb.bp.110.033431

${ }^{1}$ Liverpool University; ${ }^{2}$ Peasley Cross Hospital, St Helens, Merseyside, UK Correspondence to Emad Salib (esalib@hotmail.com)

First received 8 Nov 2010, final revision 8 May 2011, accepted 28 Jun 2011

\begin{abstract}
Aims and method To examine the association between the use of cholinesterase inhibitors (ChEls) and time to care home placement. We compared patients who were prescribed ChEls in 2006 with those who were not with respect to their placement in care homes over a 4-year period, using survival analysis.
\end{abstract}

Results During the first 30 months of follow-up there was a delay in care home placement by a median of 12 months in those who took ChEls compared with those who did not. However, at the end of the follow-up there was no significant reduction in the probability of being in a care home setting between those who had taken ChEls compared with those who had not (hazard ratio $0.75,95 \% \mathrm{Cl} 0.25-6$, $P=0.1$.

Clinical implications The study provides some evidence to suggest that prescribing ChEls may be associated with a delay in the timing of care home placement as observed in the first 2.5 years of treatment. However, based on purely observational data, no conclusion can be made as to whether such association is causal.

Declaration of interest None.
A number of observational studies have investigated the long-term effect of cholinesterase inhibitors (ChEIs) using placement in residential care as a measure of outcome. ${ }^{1-4}$ (For an analysis of the studies cited here, see online Table DS1.) These have reported that ChEIs significantly reduce the risk of admission into residential care, with a delay of up to 17.5 months being proposed. ${ }^{3}$ However, several methodological limitations significantly limit inferential value of those studies and their findings are far from conclusive. It is therefore understandable that a review of anti-dementia medications carried out by the National Institute for Health and Clinical Excellence (NICE) concluded that 'although it was clinically plausible that treatment [with ChEIs] may delay time to institutionalisation, limited direct evidence was available to assess the magnitude of this effect'. ${ }^{5}$ Previous research in this area has been carried out overseas, predominantly in the USA. The NICE review utilised this evidence, along with submissions from the manufacturers of ChEIs. Given the possibility of conflicting interests in these studies and the differences between the UK and the USA with respect to medical and residential care services, we felt a UK-based evaluation was warranted.

In this observational study based in St Helens, Merseyside, we attempt to explore whether the association between prescribing ChEIs and care home placement reported previously in the literature is also observed locally in our patient population.

\section{Method}

We retrospectively collected information about individuals who were referred to elderly psychiatric services in St Helens in 2006. Those with chronic functional illness and psycho-organic conditions with or without cognitive impairment were not included in the study. Overall, 127 individuals with a diagnosis of probable and possible Alzheimer's disease who had been prescribed ChEIs in 2006 were identified from the Cognitive Function Clinic register at Peasley Cross Hospital. The clinic register along with patient case notes provided data relating to demographics, clinical diagnosis, date of commencing treatment and name of the ChEI prescribed. Of the patients who had a diagnosis of dementia but were not prescribed a ChEI $(n=420)$, we selected $212(50 \%)$ who were considered to be a comparable control group, based on diagnostic categories and baseline Mini-Mental State Examination (MMSE) ${ }^{6}$ in 2006. Their diagnoses included senile dementia of the Alzheimer's type, early-onset dementia and dementia of combined aetiology. The degree of severity of cognitive impairment may have influenced, negatively or positively, the probability of care home placement and also survival, therefore patients with diagnoses of advanced (severe) Alzheimer's disease, vascular dementia and unspecified dementia $(n=168)$ as well as those with diagnoses of amnesic mild cognitive impairment, early dementia and alcohol-related dementia $(n=40)$ were not selected to the control group. Individuals who had an in-patient stay on our dementia ward and those with incomplete records were 
excluded from the evaluation. If a person was commenced on a ChEI but discontinued treatment before either placement in residential care or the end of the study period, they were also excluded. At 4 years' follow-up it was determined whether the patient had been placed in care or remained in their own home. If they had been admitted to a care home, date of admission was recorded. In the event of death during the review period, date and place of death were recorded.

\section{Statistical analysis}

Survival analysis was performed using STATA version 8 for Windows. The Kaplan-Meier method with log-rank test was applied to compare survival curves in the two groups. Cox proportional hazards regression assessed the effect of ChEIs on the probability of the admission into care and calculated hazard ratio (HR) with $95 \%$ confidence intervals. Hazard ratio was interpreted as the instantaneous relative risk of admission into care, at any time between 2006 and 2010, for individuals on ChEIs compared with those not on ChEIs, controlling for age, gender and deaths reported during the follow-up.

\section{Results}

A total of 339 individuals with dementia referred to St Helens psychiatric services for the elderly in 2006 were included in the analysis; 219 (65\%) were females and 120 $(35 \%)$ were males. The mean age at the time of referral was 82 years (s.d. = 7). Cholinesterase inhibitors were prescribed to 127 (23\%) patients: $74 \%$ of these were given donepezil, $14 \%$ galantamine, $8 \%$ rivastigmine and $4 \%$ memantine in combination with a ChEI. In $56 \%$ of individuals a diagnosis of probable Alzheimer's disease was made, $34 \%$ had a diagnosis of dementia of combined aetiology (possible Alzheimer's disease) and the remaining $10 \%$ had a diagnosis of Parkinson's disease-related dementia, Lewy body dementia or other dementias. Individuals who were not prescribed ChEIs had diagnoses that included senile dementia of the Alzheimer's type, early-onset dementia and dementia of combined aetiology.

The mean survival time from referral was 33 months (s.d. =13) and mean survival time in a care home was 18 months (s.d. $=12$ ).

Of those who remained at home throughout the study period (Table 1), significantly more individuals who took ChEIs were alive at the end of the 4 years compared with those who had not taken ChEIs (odds ratio (OR) $=1.9,95 \%$ CI 1.1-3.4; $P<0.01$ ). This association was also observed in people who were placed in a care home during the review period, but it did not reach statistical significance $(\mathrm{OR}=1.5$, 95\% CI 0.3-11; $P=0.07$ ).

At 4 years' follow-up, 68 individuals (20\%) had been admitted into a care home. The mean duration, in months, before placement in residential care for both males and females is shown in Table 2. The mean difference in time to admission into care between the treatment and nontreatment groups was 5 months, reaching statistical significance $(F=7$; d.f. $=1 ; P=0.04)$. This was evident in both males and females.

Figure 1 shows the proportions, with 95\% CI, of patients who at the end of the 4-year follow-up were
Table 1 Survival and placement of patients at the end of the 4-year review

\begin{tabular}{lccr} 
& \multicolumn{3}{c}{ Cholinesterase inhibitors } \\
\cline { 2 - 4 } $\begin{array}{l}\text { Placement at 4-year } \\
\text { follow-up }\end{array}$ & $\begin{array}{c}\text { Not } \\
\text { prescribed }\end{array}$ & Prescribed & Total \\
\hline Still living at home & & & \\
Survived & 130 & 87 & 217 \\
Died & 42 & 12 & 54 \\
Total & 172 & 99 & 271 \\
\hline Admitted into care & & & \\
Survived & 37 & 26 & 63 \\
Died & 3 & 2 & 5 \\
Total & 40 & 28 & 68 \\
\hline
\end{tabular}

living either at their own home or in a care home, adjusted for gender and a number of deaths within the two groups. A delay was observed in care home placements for patients who took ChEIs as compared with those who did not, but as the graph clearly shows, confidence intervals for the proportions overlapped in most periods. At 40 months, there was no observed difference between the treatment and non-treatment groups, with an equal proportion of patients in the two groups remaining at home $(\mathrm{HR}=0.75$, 95\% CI 0.25-6; $P=0.1$ ).

Figure 2 compares the observed and predicted proportions as shown in Kaplan-Meier and Cox survival curves. The difference between the observed and predicted was more evident in the patients on ChEIs in the first 2.5 years of follow-up. However, log-rank test of equality of survivor functions did not reach statistical significance $(P=0.36)$. The analysis was then restricted to patients who had been placed in care at the end of follow-up.

Figure 3 shows the proportions, with 95\% CI, of patients who were placed in care homes while taking ChEIs. For patients who took ChEIs, half of the care home placement had occurred within 27 months following referral to elderly psychiatric services. When compared with the patients who had not taken ChEIs, there was a significant delay of 12 months in median time to care home placement (Mann-Whitney $P<0.05$ ). At 20 months, care home placements included $35 \%$ of patients who had taken ChEIs and $60 \%$ of those who had not. At 30 months, the proportions were $50 \%$ for the treatment group and $80 \%$ of

\begin{tabular}{|cccc|}
\hline Table 2 Mean duration before care home placement \\
& \multicolumn{3}{c|}{ Cholinesterase inhibitors } \\
\cline { 2 - 4 } & Not & & \\
& prescribed & Prescribed & Total \\
\hline Females, $n$ & 132 & 87 & 219 \\
Still living at home, $n$ & 103 & 67 & 170 \\
Mean duration (s.d.) & $31(3)$ months & $42(3)$ months & \\
Admitted into care, $n$ & 29 & 20 & 49 \\
Mean duration (s.d.) & $35(4)$ months & $41(14)$ months & \\
\hline Males, $n$ & 80 & 40 & 120 \\
Still living at home, $n$ & 69 & 32 & 101 \\
Mean duration (s.d.) & $28(10)$ months & $42(3)$ months & \\
Admitted into care, $n$ & 11 & 8 & 19 \\
Mean duration (s.d.) & $34(5)$ months & $39(11)$ months & \\
\hline
\end{tabular}




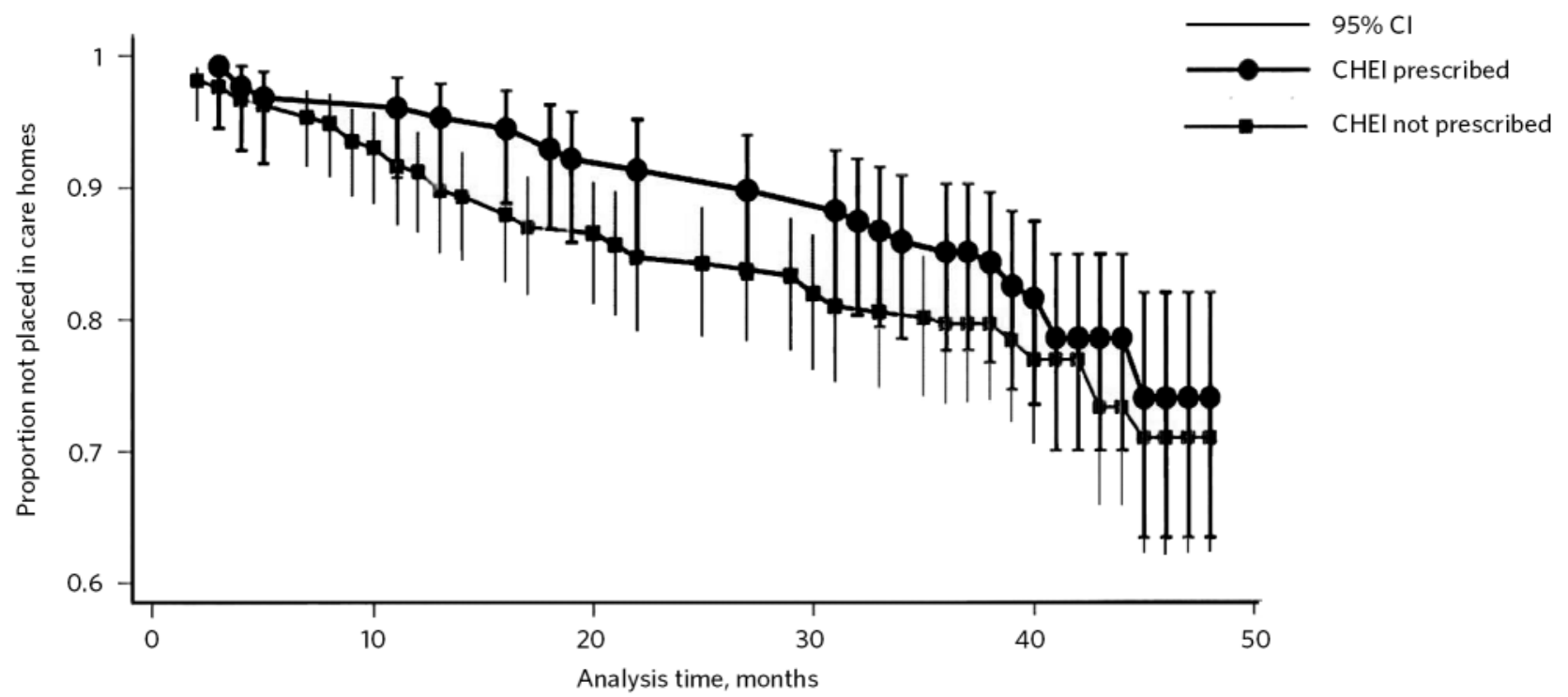

Fig 1 Proportion of care home placement for the two groups, adjusted for age, gender and number of deaths. Sample size $n=339$. ChEl, cholinesterose inhibitor.

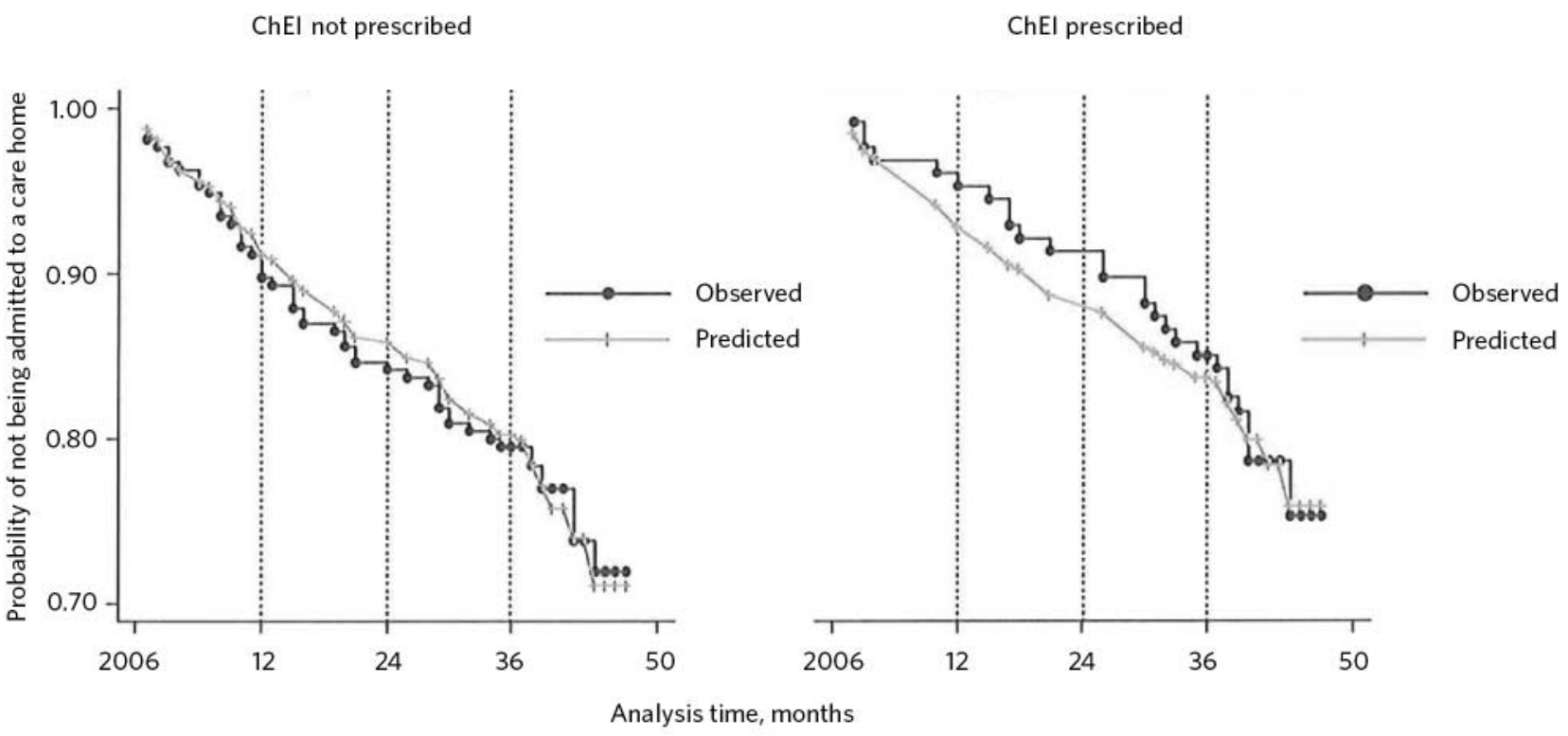

Fig 2 Observed and predicted probability of care home placement for the two groups; Kaplan-Meier and Cox survival curves compared. ChEl, cholinesterase inhibitor.

the non-treatment group $(\mathrm{HR}=0.97,95 \%$ CI $0.76-0.99)$. Log-rank test for equality of survivor function $\left(\chi^{2}=4\right.$, d.f. $=1$; $P=0.04)$ indicated a significant difference in proportions in those who were admitted into care. However, the difference in these proportions has to be carefully interpreted in view of the overlap of confidence intervals for the treatment and non-treatment groups, as illustrated in Fig. 3.

\section{Discussion}

\section{Summary of findings}

There was an observed median delay of 12 months in care home placement of patients who were prescribed ChEIs compared with those who were not during the first 2.5 years of treatment. However, survival analysis over the follow-up period, after adjusting for other variables such as gender, age and number of deaths reported, showed that at 40 months those who had taken ChEIs and those who had not had an equal probability of being placed in a care home.

\section{Methodological limitations}

We accept that a major source of bias in this review, as well as in other studies that have preceded it, is the number of potential co-variables, which may influence the decision to place in a care home. Patient variables may include initial level and rate of deterioration in cognition and functioning, as well as behavioural problems. ${ }^{7}$ Carers' ability to cope and their own medical problems, along with local availability of 


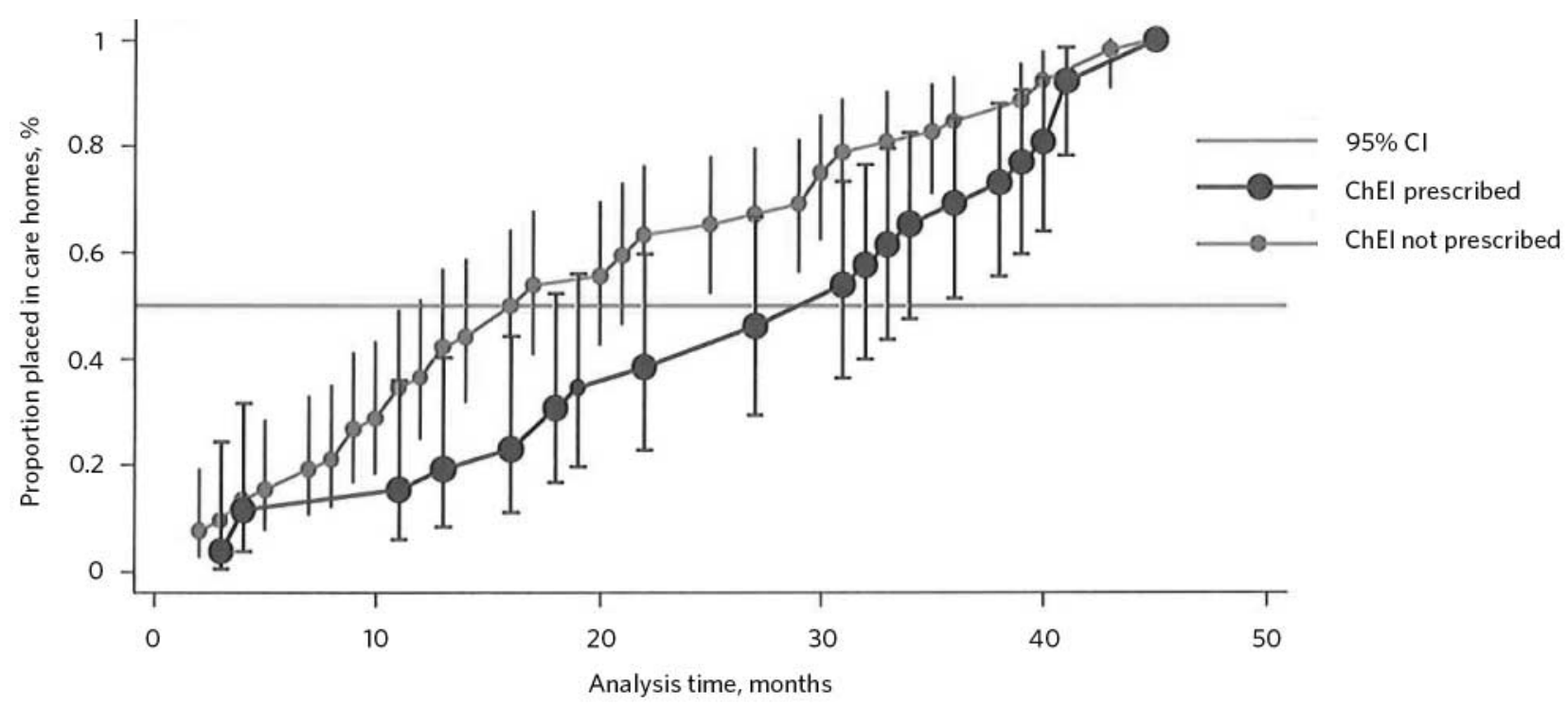

Fig 3 Care home placement at the end of follow-up. ChEl, cholinesterase inhibitor.

care home beds, day care and home care services, ${ }^{8}$ all could have potentially affected our results.

The study relied on routinely collected data obtained from the information department at the trust, the local authority database, the Cognitive Function Clinic register and patient case notes. Information bias which is expected to have occurred in both groups may have been more differential than random, especially in relation to the group who were prescribed ChEIs. Another source of bias is expected to have been the survival difference between the groups. Patients within the treatment and non-treatment groups were unmatched, which is expected to result in confounding that could have led to an over- or underestimation of the difference between the groups (i.e. other differences between the groups that may have influenced the outcome rather than taking or not taking the ChEIs). However, exploratory analysis of the data was reassuring that the comparison groups did not differ significantly despite the unmatched selection. Individuals were excluded from the analysis if they were admitted as in-patients to the dementia ward, if they had incomplete records, or if they had discontinued their ChEIs treatment for any reason. Patients with chronic functional illness, some of whom may have had a degree of cognitive impairment, as those with functional illness were also excluded. Because of missing information, we did not include in the analysis the type and dose of the ChEI used, and neither did we include other medication, medical history, physical health and socioeconomic variables that may have also confounded the findings.

\section{Interpretation of findings}

The findings should be interpreted in the light of the methodological limitations outlined above. The study provides some evidence to suggest that prescribing ChEIs may be associated with a delay in care home placement, observed in the first 2.5 years of treatment. However, based on observational data alone, no conclusion can be made as to whether such association is causal or more likely the effect of a number of factors, one of which is the prescribing of ChEIs. There is also evidence to suggest that the delayed timing of care home placement is relatively short lived, with no statistical significance after 3 years between treatment and non-treatment groups in terms of their place of residence.

We were unable to replicate the findings of Lopez et $a l^{4}$ from 2002, who reported that at 3 years from recruitment only $6 \%$ of patients treated with ChEIs were in residential care, but the proportion was $41 \%$ for those who were not treated. However, it is worth noting that the 12-month median delay in care home placement associated with ChEI treatment we have found is in keeping with the findings of Geldmacher et $a l,{ }^{3}$ who reported a median delay of 17.5 months in their double-blind randomised controlled trial. It should be stated that in our study, if the means were used, the delay associated with ChEIs would be 5 months. We felt that given the skewed distribution of data, the median estimate was probably a more reliable parameter. We were also unable to confirm the reports of Lopez et al from a later study in 2009 that ChEIs were associated with a reduced hazard ratio of 0.37 (95\% CI $0.27-0.49)$ for nursing home placement compared with the untreated group. Although our study demonstrated a delay in admission to care homes, it did not confirm that ChEIs actually reduced the overall risk of admission into care $(\mathrm{HR}=0.75,95 \% \mathrm{CI}$ $0.25-6 ; P=0.1)$.

Our finding that significantly more individuals who took ChEIs were alive at the end of the 4-year follow-up compared with those who did not take ChEIs has been previously reported. ${ }^{9}$ However, the role of confounding and bias should be assessed fully before any conclusion can be made, and this is beyond the scope of our study. The possible effect of ChEIs on patients' survival rates is an important finding and highlights another area that ought to be explored further in adequately designed studies with sufficient power. 


\section{Clinical implications}

Although the study provides some evidence to suggest that prescribing ChEIs may be associated with a delay in the timing of care home placement in the first 2.5 years of treatment, no conclusion can be made as to whether such association is causal. Moving a person to a care home is a significant and distressing event both for the individual and their family, and most carers wish to maintain home care for as long as possible. ${ }^{10}$ Being able to quantify the potential delay of care home placement associated with ChEI treatment would be of great value. As well as increasing our understanding of these drugs, it will have implications in assessing the cost-effectiveness of anti-dementia medications overall.

Any measure that may help to delay institutional care is invaluable but we must ensure that the evidence that ChEI prescribing is associated with delayed care home placement is robust and not the result of bias, confounding or chance. Despite its methodological limitations, our study provides information that may be used in the design and power calculation of future studies in different parts of the UK and elsewhere. We welcome the NICE recommendation ${ }^{5}$ for further research in this area.

\section{Acknowledgements}

We are most grateful to Ann Daniels, Sue Burrows, Jacqui Evans and the Cognitive Function Clinic team at Peasley Cross Hospital, St Helens, for their invaluable help in data collection. The authors are also grateful to Bernie Hayes and Sarah Cross, Hollins Park Library, 5 Boroughs Partnership Trust and to Mr Robert Thompson for their input.

\section{About the authors}

Emad Salib MB, MSc, MRCPI, FRCPsych, is an honorary senior lecturer at Liverpool University, and Jessica Thompson MB, ChB, is a Foundation Year
2 doctor in old age psychiatry, Peasley Cross Hospital, St Helens, Merseyside.

\section{References}

1 Lopez OL, Becker JT, Wahed AS, Saxton J, Sweet R, Wolk D, et al. Longterm effects of the contaminant use of memantine with cholinesterase inhibition in Alzheimer's disease. J Neurol Neurosurg Psychiatry 2009; 80: 600-7.

2 Feldman HH, Pirttila T, Dartigues JF, Everitt B, Van Baelen B, Schwalen S, et al. Treatment with galantamine and time to nursing home placement in Alzheimer's disease patients with and without cerebrovascular disease. Int J Ger Psychiatry 2009; 24: 479-88.

3 Geldmacher DS, Provenzano G, McRae T, Mastey V, leni JR. Donepezil is associated with delayed nursing home placement in patients with Alzheimer's disease. J Am Ger Soc 2003; 51: 937-44.

4 Lopez OL, Becker JT, Wisniewski S, Kaufer D, DeKosky ST. Cholinesterase inhibitor treatment alters the natural history of Alzheimer's disease. J Neurol Neurosurg Psychiatry 2002; 72: 310-4.

5 National Institute for Health and Clinical Excellence. Alzheimer's Disease - Donepezil, Galantamine, Rivastigmine and Memantine (Review). Appraisal Consultation Document. NICE, 2010 (http://www.nice.org.uk/ guidance/index.jsp?action=article\&o=51047).

6 Folstein MF, Folstein SE, McHugh PR. 'Mini-mental state'. A practical method for grading the cognitive state of patients for the clinician. J Psychiatr Res 1975; 12: 189-98.

7 Cohen CA, Gold DP, Shulman KI, Woetley JT, McDonald G, Wargon M. Factors determining the decision to institutionalise dementing individuals: a prospective study. Gerontologist 1993; 33: 714-20.

8 Hope T, Keene J, Gelding K, Fairburn C, Jacoby R. Predictors of institutionalisation for people with dementia living at home with a carer. Int J Ger Psychiatry 1998; 13: 682-90.

9 Gasper MC, Ott BR, Lapane KL. Is donepezil therapy associated with reduced mortality in nursing home residents with dementia? Am J Ger Pharmacotherapy 2005; 3: 1-7.

10 Karlawish JH, Klocinski JL, Merz J, Clark CM, Asch DA. Caregiver's preferences for the treatment of patients with Alzheimer's disease. Neurology 2000; 55: 1008-14. 